\title{
DIVERSITY ASSESSMENT OF BENTHIC MACROINVERTEBRATES, YEASTS, AND MICROBIOLOGICAL INDICATORS ALONG A LONGITUDINAL GRADIENT IN SERRA DO CIPÓ, BRAZIL
}

\author{
CAllisto, M., ${ }^{1}$ GOUlarT, M., ${ }^{1}$ MedeIROS, A. O., ${ }^{2}$ MORENO, P. ${ }^{1}$ and ROSA, C. A. ${ }^{2}$ \\ ${ }^{1}$ Departamento de Biologia Geral, Laboratório de Ecologia de Bentos, Universidade Federal de Minas Gerais, \\ Instituto de Ciências Biológicas, C.P. 486, CEP 30161-970, Belo Horizonte, MG, Brazil \\ ${ }^{2}$ Laboratório de Ecologia e Biotecnologia de Leveduras, Depto. Microbiologia, ICB-UFMG, Universidade Federal \\ de Minas Gerais, Instituto de Ciências Biológicas, C.P. 486, CEP 30161-970, Belo Horizonte, MG, Brazil \\ Correspondence to: Marcos Callisto, Laboratório de Ecologia de Bentos, Departamento de Biologia Geral, \\ Instituto de Ciências Biológicas, Universidade Federal de Minas Gerais, C.P. 486, CEP 30161-970, \\ Belo Horizonte, MG, Brazil, e-mail: callisto@icb.ufmg.br \\ Received April 24, 2003 - Accepted July 7, 2003 - Distributed November 30, 2004
}

(With 2 figures)

\begin{abstract}
The main goals of this study were: 1) to evaluate the structure, diversity, and functional trophic group composition of benthic macroinvertebrate communities; 2) to characterize water quality in the headwaters of the Doce river watershed, based on physical, chemical, and biological parameters (benthic macroinvertebrates, fecal coliforms, heterotrophic bacteria, and yeasts); and 3) to contribute to the knowledge of the structure and function of longitudinal gradients in lotic ecosystems in Brazil. A total of 60 benthic macroinvertebrate taxa were identified, the dominant group being the aquatic insects, with 50 families distributed in 8 orders. The dry period presented higher values of taxonomic richness and total density of benthic macroinvertebrates. A decreasing gradient was observed in these variable values from the $3^{\text {rd }}$ order stretch down to the $6^{\text {th }}$ order stretch. The highest Shannon-Wiener diversity values were found in the rainy period in the $3^{\text {rd }}$ order stretches, which presented well-developed riparian forest. Besides the $3^{\text {rd }}$ order stretches, the Pielou evenness index values were also high in the $6^{\text {th }}$ order stretch. The collectors, together with the scrapers, predominated in the benthic macroinvertebrate communities in all river stretches, except in the $2^{\text {nd }}, 4^{\text {th }}$, and $5^{\text {th }}$ order stretches in the rainy period, where communities were dominated by filterers. The shredders and predators presented low densities for all river stretches. All microbiological variables presented low levels. Due to the high counts of heterotrophic bacteria and coliforms, the studied river stretches presented inadequate potability but adequate balneability levels. The results suggest that the structure, diversity, and composition of the benthic macroinvertebrate communities are influenced by the trophic resource availability, seasonality, and sediment heterogeneity. The microbiological results of this study allow inferring that the waters from Serra do Cipó have excellent potential for recreational use and as future sources of water for human consumption.
\end{abstract}

Key words: longitudinal gradients, diversity assessment, macroinvertebrates, yeasts, microbiological indicators.

\section{RESUMO}

\section{Avaliação da diversidade de macroinvertebrados bentônicos, leveduras e indicadores microbiológicos ao longo de um gradiente longitudinal na Serra do Cipó, Brasil}

Os principais objetivos deste estudo foram: 1) avaliar a estrutura, a diversidade e a composição de grupos tróficos funcionais das comunidades de macroinvertebrados bentônicos; 2) caracterizar a qualidade das 
águas nas cabeceiras da bacia do rio Doce, com base em parâmetros físicos, químicos e biológicos (macroinvertebrados bentônicos, coliformes fecais, bactérias heterotróficas e leveduras); e 3) contribuir para o conhecimento da estrutura e do funcionamento de gradientes longitudinais em ecossistemas lóticos no Brasil. No total, 60 taxa de macroinvertebrados bentônicos foram identificados, sendo os insetos aquáticos o grupo dominante, com 50 famílias distribuídas em 8 ordens. O período de seca apresentou os maiores valores de riqueza taxonômica e de densidade total de macroinvertebrados bentônicos. Foi observado gradiente decrescente nos valores dessas variáveis do trecho de $3^{\mathrm{a}}$ ordem ao trecho de $6^{\mathrm{a}}$ ordem. Os maiores valores do índice de diversidade de Shannon-Wiener foram encontrados no período de chuvas nos trechos de $3^{\underline{a}}$ ordem, que apresentaram mata ciliar bem desenvolvida. Além dos trechos de $3^{\underline{a}}$ ordem, os valores do índice de eqüitabilidade de Pielou também foram elevados no trecho de $6^{a}$ ordem. Os coletores, juntamente com os raspadores, predominaram nas comunidades de macroinvertebrados bentônicos, exceto nos trechos de $2^{\mathrm{a}}$, 4 $4^{\mathrm{a}}$ e $5^{\mathrm{a}}$ ordens, durante o período de chuvas, quando houve o domínio de filtradores. Os fragmentadores apresentaram baixas densidades na maioria dos trechos de rio estudados. Todas as variáveis microbiológicas apresentaram baixos valores. Os trechos de rio estudados são impróprios quanto à potabilidade e próprios para balneabilidade, em decorrência das altas contagens de bactérias heterotróficas e coliformes. Os resultados sugerem que a estrutura, a diversidade e a composição das comunidades de macroinvertebrados bentônicos são influenciadas pela disponibilidade de recursos tróficos, pela sazonalidade e pela heterogeneidade do sedimento. Os resultados microbiológicos nos permitem inferir que as águas da Serra do Cipó apresentam potencial para uso recreacional e como fonte futura de água para abastecimento humano.

Palavras-chave: gradientes longitudinais, avaliação da diversidade, macroinvertebrados, leveduras, indicadores microbiológicos.

\section{INTRODUCTION}

Within recent decades aquatic ecosystems have been altered at different scales, and registered as negative consequences of anthropogenic activities (e.g., mining, dam construction, artificial eutrophication, river canalization, and recreation). Detection of resulting impacts on streams depends on the use of biomonitors combined with physical (e.g., temperature, suspended solids) and chemical (e.g., nutrient levels, concentrations of potential toxins) data (Dudgeon, 1994).

Biological indicator use in monitoring programs provides a more exact measure of anthropogenic effects on aquatic ecosystems (Callisto \& Esteves, 1995; Callisto et al., 2001a). Biological indicators have the advantage of monitoring water quality over a long period of time, thus providing a more adequate picture of level of pollutant effects on the ecosystem than is the case for chemical methods, which provide only momentary evidence of water quality (Tundisi \& Barbosa, 1995).

Within the organisms commonly used as biological indicators, benthic macroinvertebrates stand out as ideal due to: relatively low mobility and long life cycles, reflecting temporal patterns and local conditions; high diversity, abundance and, consequently, in providing a wide range of responses to different environmental pollution agents; large size and easy identification at high taxonomic (such as family) resolution by non-specialists; well standardized and low-cost methodologies; and temporal and spatial stability, reflecting changes in ecosystem processes (Rosenberg \& Resh, 1993).

Microbiological monitoring of organisms important in determining water contamination levels is usually done by fecal pollution indicators, represented by bacteria counts of coliform groups (Ceballos et al., 1995; Lutterback et al., 2001). This parameter is used by government agencies to classify water bodies as to use and sanitary levels (balneability and potability). Aquatic bacteria and fungi feed on dissolved organic matter, multiplying rapidly under favorable conditions. Some authors suggest that the number and composition of yeast species present in rivers and lakes can be used as organic enrichment indicators in water bodies (Rosa et al., 1995; Morais et al., 1996). Species within the genera Cryptococus, Debaryomyces, and Rhodotora are characteristically found in nonpolluted waters, while Candida and Saccharomyces species can be frequently found in eutrophic waters (Hagler et al., 1986; Rosa et al., 1995). 
The main goals of this study were: 1) to evaluate the structure, diversity, and functional trophic group composition of benthic macroinvertebrate communities; 2 ) to characterize the water quality in the headwaters of the Doce river watershed, based on physical, chemical, and biological parameters (benthic macroinvertebrates, fecal coliforms, heterotrophic bacteria and yeasts); and 3) to contribute to knowledge of longitudinal gradient structure and function in lotic ecosystems in Brazil.

\section{Study area}

Located south of the Espinhaço Cordillera (19 ${ }^{\circ}$ $20^{\circ} \mathrm{S}$ and $43^{\circ}-44^{\circ} \mathrm{W}$ ) in the center of Minas Gerais State, Serra do Cipó is a watershed of the Doce river and São Francisco river basins. The vegetation is composed of tropical savanna (cerrado) at the lower altitudes (700-900 $\mathrm{m}$ a.s.1.), rupestrian fields in the highest portions (above 1,200 $\mathrm{m}$ a.s.l.), and riparian forest in the humid valleys along the rivers. The climate is classified as Cwb (Köppen), with rainy summers and dry winters, and presents an annual mean pluviosity of 1,500 mm/year (Galvão \& Nimer, 1965). The longitudinal gradient was studied in the Indaiá stream ( $1^{\text {st }}$ up to $4^{\text {th }}$ orders) and Peixe river $\left(5^{\text {th }}\right.$ and $6^{\text {th }}$ orders), both belonging to the Doce river watershed.

\section{MATERIAL AND METHODS}

\section{Physical and chemical characterization of the waters}

Chemical and physical variables measured with a Horiba multiprobe were: depth (m), temperature $\left({ }^{\circ} \mathrm{C}\right), \mathrm{pH}$, electrical conductivity $(\mu \mathrm{S} / \mathrm{cm})$, and dissolved oxygen $(\mathrm{mg} / \mathrm{L})$. Concentrations of total phosphorus, orthophosphate, ammonium, nitrate/ nitrite, total nitrogen, and reactive soluble silica were determined in the Laboratory of Limnology (Institute of Biological Science, Federal University of Minas Gerais), using water samples collected bimonthly in the water sub-surface, according to Golterman et al. (1978) and Mackereth et al. (1978). The total alkalinity was determined by the Gran method, modified by Carmouze (1994).

\section{Ecological characteristics of watershed stretches}

To evaluate ecologically the sampling stations and their surroundings we used a rapid evaluation protocol of ecological conditions and habitat diver- sity in watershed stretches, proposed by Callisto $e t$ al. (2002). This protocol evaluates not only freshwater environments but also land use and occupation of surrounding areas of drainage basins. The major variables assessed were river width and depth (m); riparian vegetation composition; dominant fraction of organic matter (coarse/fine particulate organic matter as proposed by Ward, 1992); habitat diversity (riffles/pools); sediment composition (bedrock, cobbles, pebbles, gravel, sand). The water current velocity was measured using a Global Water digital fluxmeter.

\section{Benthic macroinvertebrate communities}

Samplings were performed during the rainy (February) and dry (June) periods of 2000, along a longitudinal gradient in the Indaiá stream $\left(1^{\text {st }}\right.$ up to $4^{\text {th }}$ order stretches) and Peixe River $\left(5^{\text {th }}\right.$ and $6^{\text {th }}$ order stretches). Samples were collected using Surber samplers $(0.250 \mathrm{~mm}$ mesh $)$ from a $0.0625 \mathrm{~m}^{2}$ area on the gravel/sand substrate, submerged leaves/ branches of the riparian vegetation substrate; and $0.01 \mathrm{~m}^{2}$ from remaining substrates. The samples were then immediately fixed with $10 \%$ formaline. A total of 120 samples were collected (60 samples in each sampling period) (Table 1).

In the laboratory, the samples were washed using $0.250 \mathrm{~mm}$ sieves, sorted and, under stereomicroscope and with the use of appropriate literature (Dominguez et al., 2001; Pérez, 1988; Wiggins, 1977), identified. Identified organisms were preserved in $70 \%$ ethanol and deposited in the Reference Collection of Benthic Macroinvertebrates at the Institute of Biological Sciences, Federal University of Minas Gerais, according to Callisto et al. (1998).

Functional feeding classification of the benthic macroinvertebrate communities was performed according to Domínguez et al. (2001), Epler (2001), Cranston (1996), and Pescador (1997). The relative dominance of the main trophic groups was estimated taking into consideration shredders, collectors, filterers, scrapers, and predators (Callisto et al., 2001b).

Taxonomic richness was preferentially recorded at the family level, and the evenness and diversity indexes were calculated for each sample period, river stretch, and basin using the ShannonWiener diversity index and Pielou evenness index (Magurran, 1991). 
TABLE 1

River stretches, riparian vegetation type, sampled ecosystem and respective sampled substrates. *IS = Indaiá stream; PS = Peixe river in a Sobrado farm locality; $\mathbf{P C}=$ Peixe river, confluence with Preto do Itambé river.

\begin{tabular}{|c|c|c|c|}
\hline $\begin{array}{c}\text { River } \\
\text { stretch }\end{array}$ & $\begin{array}{c}\text { Riparian } \\
\text { vegetation }\end{array}$ & Ecosystem & Sampled substrate \\
\hline $1^{\text {st }}$ order & Rupestrian fields & IS & Filamentous algae \\
\hline $1^{\text {st }}$ order & Rupestrian fields & IS & Deposit of fine detritus \\
\hline $2^{\text {nd }}$ order & Rupestrian fields & IS & Algae biofilm \\
\hline $2^{\text {nd }}$ order & Rupestrian fields & IS & Moss banks \\
\hline $2^{\text {nd }}$ order & Rupestrian fields & IS & Gravel and sand deposits \\
\hline $3^{\text {rd }}$ order & $\begin{array}{l}\text { Riparian forest, } \\
\text { closed canopy }\end{array}$ & IS & Moss banks \\
\hline $3^{\text {rd }}$ order & $\begin{array}{l}\text { Riparian forest, } \\
\text { closed canopy }\end{array}$ & IS & Gravel and sand deposits \\
\hline $3^{\text {rd }}$ order & $\begin{array}{l}\text { Riparian forest, } \\
\text { open canopy }\end{array}$ & IS & Algae biofilm \\
\hline $3^{\text {rd }}$ order & $\begin{array}{l}\text { Riparian forest, } \\
\text { open canopy }\end{array}$ & IS & Moss banks \\
\hline $3^{\text {rd }}$ order & $\begin{array}{l}\text { Riparian forest, } \\
\text { open canopy }\end{array}$ & IS & Gravel and sand deposits \\
\hline $4^{\text {th }}$ order & Rupestrian fields & IS & Algae biofilm \\
\hline $4^{\text {th }}$ order & Rupestrian fields & IS & Moss banks \\
\hline $4^{\text {th }}$ order & Rupestrian fields & IS & Gravel and sand deposits \\
\hline $4^{\text {th }}$ order & Rupestrian fields & IS & Eriocaulaceae banks \\
\hline $5^{\text {th }}$ order & $\begin{array}{c}\text { Secondary } \\
\text { forest, pastures }\end{array}$ & PS & Algae biofilm \\
\hline $5^{\text {th }}$ order & $\begin{array}{c}\text { Secondary } \\
\text { forest, pastures }\end{array}$ & PS & Moss banks \\
\hline $5^{\text {th }}$ order & $\begin{array}{c}\text { Secondary } \\
\text { forest, pastures }\end{array}$ & PS & Gravel and sand deposits \\
\hline $5^{\text {th }}$ order & $\begin{array}{c}\text { Secondary } \\
\text { forest, pastures }\end{array}$ & PS & Submerged angiosperms \\
\hline $5^{\text {th }}$ order & $\begin{array}{l}\text { Secondary } \\
\text { forest, pastures }\end{array}$ & $\mathrm{PC}$ & Deposit of fine detritus \\
\hline $6^{\text {th }}$ order & $\begin{array}{c}\text { Secondary } \\
\text { forest, pastures }\end{array}$ & $\mathrm{PC}$ & $\begin{array}{l}\text { Leaves and branches of } \\
\text { marginal vegetation }\end{array}$ \\
\hline
\end{tabular}

\section{Microbiological indicators}

For microbiologic analyses, sub-surface water samples were immediately put into sterile glass bottles and returned to the laboratory on ice within 8 hours for processing. Total and fecal coliforms were determined using the standard most probable number method (MNP); heterotrophic bacteria counts were performed using the pour plate method on NWRI agar-HPCA (peptone $0.3 \%$, soluble casein $0.05 \%$, $\mathrm{K}_{2} \mathrm{HPO}_{4} 0.02 \%, \mathrm{MgSO}_{4} 0.005 \%, \mathrm{FECL}_{3} 0.0001 \%$, agar $1.5 \%$ ) after $2-5$ days incubation at $22 \%-28^{\circ} \mathrm{C}$
(Greenberg et al., 1998). For yeast counts (CFU), aliquots of $0.1 \mathrm{ml}$ of the samples were plated in triplicates on YM agar with antibiotic (glucose 2.0\%, peptone $1.0 \%$, yeast extract $0.3 \%$, malt extract $0.3 \%$, agar $2.0 \%$, and cloramphenicol $20 \mathrm{mg} \%$ ). Plates were incubated at room temperature and counts were obtained after 3 to 5 days. Each morphological yeast biotype was counted and representative colonies were isolated, purified, and characterized according to standard methods (Yarrow, 1998). Yeasts were identified by keys in Kurtzman \& Fell (1998). 


\section{RESULTS}

\section{Water physical and chemical characteristics}

The studied stretches presented acid to slightly neutral waters, with $\mathrm{pH}$ varying between 4.2 in the $2^{\text {nd }}$ order stretch (rainy period) and 7.3 in the $5^{\text {th }}$ and $6^{\text {th }}$ order stretches (dry period), and high dissolved oxygen concentrations $\left(6.7 \mathrm{mg} / \mathrm{L}\right.$ in the $3^{\text {rd }}$ order stretch, closed canopy section in the dry period, and up to $9.1 \mathrm{mg} / \mathrm{L}$ in the $5^{\text {th }}$ order stretch, rainy period). Low electrical conductivity values $(<15 \mu \mathrm{S} / \mathrm{cm}$ in the dry period and $<25 \mu \mathrm{S} / \mathrm{cm}$ in the rainy period) and low water column turbidity (0 NTU to 34 NTU) were found. Temperature varied along the gradient, with higher values in the rainy period in stretches presenting open riparian vegetation (composed by rupestrian fields or degraded by pastures) and lower values in the well-developed riparian forest stretches (in the $3^{\text {rd }}$ order stretches) (Table 2).

Low total-phosphorus concentrations were found, varying between $1.325 \mu \mathrm{g} / \mathrm{L}$ ( $1^{\text {st }}$ order stretch, rainy period) up to $27.95 \mu \mathrm{g} / \mathrm{L}$ ( $3^{\text {rd }}$ order stretch, open canopy, dry period). Higher total nitrogen concen- trations were found in the rainy periods. The predominant ion form was ammonium-nitrogen $\left(\mathrm{NH}_{4}^{+}\right)$ (10.38-84.54 $\mu \mathrm{g} / \mathrm{L})$. Silica concentrations were relatively high $(0.14-1.50 \mathrm{mg} / \mathrm{L})$ (Table 2$)$, reflecting the quartzite soil type found in the region.

\section{Assessment of the ecological characteristics of basin stretches}

Along the longitudinal gradient in the Indaiá stream an increase in the mean river width from the $1^{\text {st }}$ up to the $6^{\text {th }}$ order was observed. On the other hand, the depth varied as a result of terrain unevenness and channel structure (Table 2). Riparian vegetation was composed of rupestrian field in the $1^{\text {st }}, 2^{\text {nd }}$, and $4^{\text {th }}$ order stretches. In $3^{\text {rd }}$ order stretches, this vegetation type was replaced by riparian forest, while in $5^{\text {th }}$ and $6^{\text {th }}$ order stretches, isolated secondary forest fragments and large pastures were seen. The fine particulate organic matter (FPOM) source followed the distribution of riparian vegetation and presence of aquatic macrophytes. In $3^{\text {rd }}$ order stretches, coarse particulate organic matter was the dominant form (CPOM), while in the remaining stretches FPOM predominated.

TABLE 2

Abiotic data obtained along the longitudinal gradient in the Serra do Cipó during the rainy and dry periods of 2000. ND: not detectable, rainy-dry seasons.

\begin{tabular}{|c|c|c|c|c|c|c|c|}
\hline \multirow{2}{*}{ Variables } & \multicolumn{7}{|c|}{ River stretches } \\
\hline & $1^{\text {st }}$ order & $2^{\text {nd }}$ order & $3^{\text {rd }} \operatorname{order}(\mathrm{A})$ & $3^{\text {rd }}$ order (B) & $4^{\text {th }}$ order & $5^{\text {th }}$ order & $6^{\text {th }}$ order \\
\hline Latitude & $19^{\circ} 16.6 \mathrm{~S}$ & $19^{\circ} 16.6 \mathrm{~S}$ & $19^{\circ} 16.4 \mathrm{~S}$ & $19^{\circ} 16.4 \mathrm{~S}$ & $19^{\circ} 16.0 \mathrm{~S}$ & $19^{\circ} 19.5 \mathrm{~S}$ & $19^{\circ} 17.6 \mathrm{~S}$ \\
\hline Longitude & $43^{\circ} 31.5 \mathrm{~W}$ & $43^{\circ} 31.5 \mathrm{~W}$ & $43^{\circ} 31.2 \mathrm{~W}$ & $43^{\circ} 31.2 \mathrm{~W}$ & $43^{\circ} 10.9 \mathrm{~W}$ & $43^{\circ} 20.9 \mathrm{~W}$ & $43^{\circ} 15.5 \mathrm{~W}$ \\
\hline Altitude (m a.s.l.) & 1,650 & 1,630 & 1,450 & 1,430 & 1,380 & 890 & 650 \\
\hline Width $(\mathrm{m})$ & 0.5 & 3.0 & 3.0 & 2.5 & 7.0 & 16.0 & 25.0 \\
\hline Depth (m) & 0.08 & 0.25 & 0.63 & 0.26 & 0.64 & 0.43 & 1.15 \\
\hline Temperature $\left({ }^{\circ} \mathrm{C}\right)$ & $22.7-19.0$ & $23.4-17.0$ & $21.5-14.0$ & $21.3-14.0$ & $21.2-14.0$ & $24.7-15.0$ & $24.3-19.0$ \\
\hline Turbidity (NTU) & 23 & 34 & 31 & 16 & 27 & 0 & 18 \\
\hline Electrical conductivity $(\mu \mathrm{S} / \mathrm{cm})$ & $23-10$ & $15-8$ & $13-5$ & $14-9$ & $10-5.59$ & $12-14$ & $14-14$ \\
\hline $\mathrm{pH}$ & $4.3-4.7$ & $4.2-6.2$ & $4.5-6.1$ & $4.4-6.4$ & $4.4-6.9$ & $5.5-7.3$ & $5.8-7.3$ \\
\hline Dissolved oxygen $(\mathrm{mg} / \mathrm{L})$ & 7.9-6.9 & $8.3-6.9$ & 7.1-6.7 & 7.4-7.0 & $8.4-6.8$ & $9.1-7.5$ & $8.7-7.5$ \\
\hline Total alkalinity $(\mu \mathrm{Eq} \mathrm{CO} / / \mathrm{L})$ & ND-ND & ND-23.5 & ND-46.5 & ND-40.7 & ND-ND & $92.2-121.3$ & $122.7-100.1$ \\
\hline Total P & $1.325-24.65$ & $9.503-19.97$ & $14.5-20.25$ & $13.34-27.95$ & $12.33-15.20$ & $13.55-14.52$ & $15.88-8.492$ \\
\hline Total $\mathrm{N}$ & $493-432.4$ & $589.9-264.7$ & $234-145.1$ & $411.5-203.6$ & $367.8-230.5$ & $244.6-241.2$ & $516.45-560.1$ \\
\hline $\mathrm{PO}_{4}^{-}$ & $9.687-2.484$ & $7.957-2.894$ & $3.057-2.134$ & $9.124-5.318$ & $3.256-11.55$ & $4.184-4.790$ & $5.885-2.904$ \\
\hline $\mathrm{NH}_{4}^{-}$ & 10.382-ND & ND-28.70 & $48.00-15.03$ & $84.54-15.05$ & $51.32-75.49$ & 26.00-ND & $14.70-34.54$ \\
\hline $\mathrm{NO}_{2}^{-}$ & ND-ND & ND-0.785 & ND-ND & ND-ND & ND-ND & ND-ND & ND-ND \\
\hline $\mathrm{NO}_{3}^{-}$ & $4.326-11.00$ & $29.00-$ & $5.193-4.994$ & $38.75-36.50$ & $35.00-30.03$ & $30.06-56.38$ & $66.75-45.40$ \\
\hline Reactive soluble silica (mg/L) & $0.926-0.318$ & $0.421-0.424$ & $0.633-0.320$ & $0.494-0.282$ & $0.365-0.144$ & $0.410-0.292$ & $0.677-1.503$ \\
\hline $\mathrm{CPOM}(\%)$ & 1 & 10 & 70 & 50 & 10 & 15 & 5 \\
\hline FPOM $(\%)$ & 99 & 90 & 30 & 50 & 90 & 85 & 95 \\
\hline Balneability level & Appropriate & Appropriate & Appropriate & Appropriate & Appropriate & Appropriate & Appropriate \\
\hline Potability level & Inadequate & Inadequate & Inadequate & Inadequate & Inadequate & Inadequate & Inadequate \\
\hline
\end{tabular}


Regarding sediment composition, riffle/pool sequences, and river channel morphology, the river stretches were grouped into four types by an ecological conditions characterization protocol (Callisto et al., 2002):

- $\quad \mathbf{1}^{\text {st }}$ order stretch: bottom formed by $100 \%$ bedrock; the stream is constituted by a small water thread during rainy period, while in the dry period, isolated pools with large FPOM deposits are formed;

- $2^{\text {nd }}$ and $4^{\text {th }}$ order stretches: bottom of over $70 \%$ bedrock; riffle/pool sequences welldefined, with riffles formed by steep slopes, and deep pools having gravel and coarse sand deposits near the river margins;

- $3^{\text {rd }}$ order stretches: bottom composed mainly by rocks, cobbles, and pebbles, with bedrock in the pools; riffle/pool sequences scattered, with short riffle stretches and long, shallow pools;

- $5^{\text {th }}$ order stretch: bottom of rocks, cobbles, pebbles, gravel, and sand; riffle/pool sequences are substituted by erosion/deposi- tional zones, with erosion zones located mainly in the river channel, and deposition zones along the margins;

- $6^{\text {th }}$ order stretch: bottom of bedrocks covered with gravel, sand, and silt deposits throughout the channel extension; the riffle/ pool sequences are substituted by erosion/ depositional zones, with erosion and depositional zones located at the river curves.

\section{Benthic macroinvertebrate communities}

Sixty macroinvertebrate benthic taxa were identified, the dominant group being the aquatic insects, with 50 families distributed in 8 orders. Taxonomic richness varied largely along the gradient, with lower values in the rainy period and a decreasing gradient from the $3^{\text {rd }}$ order (open canopy) down to the $6^{\text {th }}$ order stretch (Fig. 1). The highest taxonomic richness values were found in the dry period in the $2^{\text {nd }}$ (42 taxa) and $3^{\text {rd }}$ order (open canopy, 43 taxa) stretches, while the $6^{\text {th }}$ order stretch presented the lowest taxonomic richness values (19 taxa) (Table 3).

TABLE 3

Macroinvertebrates densities $\left(\mathrm{ind} / \mathrm{m}^{2}\right.$ ) along the longitudinal gradient in the Serra do Cipó during the rainy and dry periods of 2000 .

\begin{tabular}{|c|c|c|c|c|c|c|c|c|c|c|c|c|c|c|}
\hline \multirow{3}{*}{ Taxa } & \multicolumn{14}{|c|}{ River stretches } \\
\hline & \multicolumn{2}{|c|}{$1^{\text {st }}$ order } & \multicolumn{2}{|c|}{$2^{\text {nd }}$ order } & \multicolumn{2}{|c|}{$3^{\text {rd }}(\mathrm{A})$ order } & \multicolumn{2}{|c|}{$3^{\text {rd }}(\mathrm{B})$ order } & \multicolumn{2}{|c|}{$4^{\text {th }}$ order } & \multicolumn{2}{|c|}{$5^{\text {th }}$ order } & \multicolumn{2}{|c|}{$6^{\text {th }}$ order } \\
\hline & Rainy & Dry & Rainy & Dry & Rainy & Dry & Rainy & Dry & Rainy & Dry & Rainy & Dry & Rainy & Dry \\
\hline \multicolumn{15}{|l|}{ Diptera } \\
\hline Chironomidae & 6467 & 6867 & 7933 & 8352 & 1687 & 3213 & 2989 & 6605 & 9557 & 22397 & 15787 & 23593 & 1299 & 10037 \\
\hline Simuliidae & 100 & 133 & 14503 & 33 & 1 & - & 1111 & 1843 & 22003 & 2335 & 171307 & 29825 & 5 & 161 \\
\hline Ceratopogonidae & 67 & 33 & 3 & 48 & 1 & 243 & 3 & 33 & 16 & 156 & 49 & 121 & 71 & 111 \\
\hline Tipulidae & 33 & - & 3 & 3 & 19 & - & 25 & 176 & 9 & 3 & - & 19 & - & - \\
\hline Culicidae & - & 833 & - & - & - & - & - & 1 & - & - & - & 33 & - & - \\
\hline Empididae & - & - & - & - & - & - & - & - & 0 & 67 & 167 & 21 & - & - \\
\hline Tabanidae & - & - & - & - & - & 3 & - & - & - & - & 44 & - & - & 50 \\
\hline \multicolumn{15}{|l|}{ Ephemeroptera } \\
\hline Baetidae & 3000 & 233 & 2284 & 739 & 33 & 192 & 3935 & 1617 & 7481 & 8403 & 4993 & 19361 & 848 & 2105 \\
\hline Leptophlebiidae & 467 & 67 & 437 & 2365 & 781 & 4547 & 671 & 4216 & 533 & 2089 & 88 & 196 & 144 & 105 \\
\hline Leptohyphidae & - & - & 3 & 33 & 800 & 500 & 785 & 88 & 475 & 275 & 3421 & 5302 & 621 & 16 \\
\hline Caenidae & - & - & 8 & 20 & - & 63 & 1 & 5 & - & 24 & - & - & - & - \\
\hline Polymitarcyidae & - & - & 5 & 25 & - & 7 & - & 3 & 1 & 1 & - & - & 5 & - \\
\hline Euthyplociidae & - & - & 21 & 37 & - & 11 & 7 & 9 & 0 & 1 & - & - & - & - \\
\hline \multicolumn{15}{|l|}{ Plecoptera } \\
\hline Perlidae & - & - & 5 & 9 & 35 & 8 & 1 & 4 & 64 & 149 & - & 1 & - & - \\
\hline Gripopterygidae & - & - & - & - & - & - & - & - & 67 & - & - & 33 & 5 & - \\
\hline Trichoptera & & & & & & & & & & & 16 & & 133 & \\
\hline Helicopsychidae & - & 67 & 401 & 165 & 192 & 116 & 348 & 108 & 69 & 588 & 93 & 12 & - & - \\
\hline Polycentropodidae & - & 67 & - & 1 & 1 & 1 & 69 & 11 & - & 3 & - & - & - & 69 \\
\hline Hydrobiosidae & 33 & - & 201 & 201 & - & 4 & 133 & 33 & 167 & 100 & - & 67 & - & - \\
\hline Hydroptilidae & 33 & - & 1 & 1 & - & - & - & 108 & 236 & 800 & 496 & 2105 & - & - \\
\hline Hydropsychidae & - & 33 & 2435 & 403 & - & 136 & 35 & 147 & 2833 & 6267 & 1400 & 2332 & - & 201 \\
\hline
\end{tabular}


TABLE 3 (Continued.)

\begin{tabular}{|c|c|c|c|c|c|c|c|c|c|c|c|c|c|c|}
\hline \multirow{3}{*}{ Taxa } & \multicolumn{14}{|c|}{ River stretches } \\
\hline & \multicolumn{2}{|c|}{$1^{\text {st }}$ order } & \multicolumn{2}{|c|}{$2^{\text {nd }}$ order } & \multicolumn{2}{|c|}{$3^{\text {rd }}(\mathrm{A})$ order } & \multicolumn{2}{|c|}{$3^{\text {rd }}(\mathbf{B})$ order } & \multicolumn{2}{|c|}{$4^{\text {th }}$ order } & \multicolumn{2}{|c|}{$5^{\text {th }}$ order } & \multicolumn{2}{|c|}{$6^{\text {th }}$ order } \\
\hline & Rainy & Dry & Rainy & Dry & Rainy & Dry & Rainy & Dry & Rainy & Dry & Rainy & Dry & Rainy & Dry \\
\hline Leptoceridae & - & - & 7368 & 5172 & 36 & 20 & 100 & 120 & 5736 & 3507 & 67 & 101 & - & 111 \\
\hline Odontoceridae & - & - & 7 & 8 & 268 & 68 & 1043 & 29 & 4 & 9 & - & - & - & - \\
\hline Calamoceratidae & - & - & - & 1 & - & 1 & - & - & - & - & - & - & - & - \\
\hline Glossosomatidae & - & - & - & - & - & - & 8 & - & - & - & 244 & 33 & - & - \\
\hline Xyphocentridae & - & - & - & - & - & - & - & 1 & - & - & - & - & - & - \\
\hline \multicolumn{15}{|l|}{ Megaloptera } \\
\hline Corydalidae & - & - & 33 & 3 & 3 & 1 & 44 & 17 & 236 & 39 & - & 75 & - & - \\
\hline \multicolumn{15}{|l|}{ Heteroptera } \\
\hline Corixidae & 2033 & 900 & 39 & 63 & 4 & 3 & - & 9 & 4 & - & - & - & - & - \\
\hline Veliidae & 167 & 717 & - & 5 & - & - & 3 & - & - & 1 & - & 40 & - & - \\
\hline Pleidae & 33 & - & - & - & - & 33 & 67 & 1 & - & - & - & - & - & - \\
\hline Naucoridae & - & 200 & 15 & 24 & 203 & 331 & 711 & 228 & 287 & 161 & 49 & 21 & 199 & 11 \\
\hline Notonectidae & - & - & 1 & 16 & 3 & 8 & - & 5 & - & - & - & - & - & - \\
\hline Nepidae & - & - & - & - & - & - & - & - & - & - & - & - & 5 & - \\
\hline \multicolumn{15}{|l|}{ Odonata } \\
\hline Coenagrionidae & 33 & 33 & 24 & 79 & 40 & 149 & 51 & 55 & 69 & 20 & - & 103 & 11 & - \\
\hline Libellulidae & 33 & 33 & - & 1 & 1 & 33 & 3 & 1 & 0 & 8 & 149 & 984 & - & - \\
\hline Aeshnidae & - & - & 3 & - & 3 & - & - & - & - & - & - & - & - & - \\
\hline Megapodagrioni & - & - & - & 1 & - & 11 & - & 3 & - & - & - & - & - & - \\
\hline Calopterygidae & 33 & - & - & - & - & - & - & - & 0 & 33 & - & 33 & 43 & - \\
\hline Gomphidae & - & - & - & - & - & - & - & 1 & 0 & 3 & - & 5 & - & - \\
\hline Coleoptera & & & & & & & & & & & 59 & & & \\
\hline Psephenidae & 33 & - & 15 & 48 & 88 & 43 & 161 & 169 & 52 & 53 & - & 4 & 2 & - \\
\hline Dytiscidae & 167 & 1350 & 1 & 48 & 39 & 4 & 16 & 3 & 5 & 1 & - & 137 & - & 139 \\
\hline Noteridae & 100 & - & - & - & 1 & - & 33 & - & - & - & - & - & - & - \\
\hline Hydroscaphidae & 1600 & 867 & 3 & 8 & - & 133 & - & 53 & - & 43 & - & 92 & - & 50 \\
\hline Elmidae & - & 300 & 8640 & 1455 & 2012 & 896 & 1985 & 471 & 3661 & 16063 & 867 & 787 & 71 & 2051 \\
\hline Dryopidae & - & 200 & - & - & - & 133 & - & - & 67 & 36 & - & - & - & - \\
\hline Hydrophilidae & - & 100 & 101 & 68 & 33 & 79 & 33 & 48 & 36 & 209 & - & 2672 & 2 & - \\
\hline Gyrinidae & - & - & 5 & 3 & 1 & - & 1 & - & - & - & - & - & - & - \\
\hline \multicolumn{15}{|l|}{ Coleoptera } \\
\hline Hydraenidae & - & - & 233 & - & - & - & - & - & 319 & 12 & - & - & - & - \\
\hline Chrysomeliidae & - & - & - & 1 & - & - & - & - & - & - & - & - & - & - \\
\hline Ptilodactylidae & - & - & - & - & - & - & 1 & - & - & - & - & - & - & - \\
\hline Scirtidae & - & - & - & - & - & - & - & 1 & - & - & - & - & - & - \\
\hline Lepidoptera & - & - & 400 & 33 & - & - & 203 & 36 & 467 & 33 & 233 & 72 & 5 & \\
\hline Hidracarina & 300 & 167 & 268 & 107 & 104 & 5 & 73 & 23 & 33 & 333 & 100 & 225 & 33 & 255 \\
\hline Oligochaeta & 33 & 667 & 12 & 169 & 99 & 321 & 13 & 108 & 1 & 548 & 96 & 3051 & 469 & 401 \\
\hline Hiridunea & - & - & - & 8 & - & - & - & 4 & - & - & - & - & - & - \\
\hline Cladocera & - & - & - & 1 & - & - & - & 56 & - & - & - & - & - & - \\
\hline Copepoda & - & - & 1 & 8 & - & - & - & 11 & - & - & - & - & - & - \\
\hline Conchostraca & - & - & - & 1 & - & - & - & - & - & - & - & - & - & - \\
\hline Ostracoda & - & - & - & - & - & - & - & 5 & - & - & - & 1 & - & - \\
\hline Hydrozoa & - & - & - & - & - & - & - & 808 & - & 4 & - & 1 & - & 21 \\
\hline Bivalvia & & & & & & & & & & & & & & 5 \\
\hline \multicolumn{15}{|l|}{ Gastropoda } \\
\hline Ancylidae & - & - & - & 35 & - & - & - & - & - & - & - & - & - & - \\
\hline Thiaridae & - & - & - & - & - & - & - & - & - & - & - & - & - & 111 \\
\hline Total density & 14,767 & 13,867 & 45,413 & 19,804 & 6,488 & 11,316 & 14,663 & 17,276 & 64,723 & 60,356 & 45,528 & 91,380 & 3,972 & 11,337 \\
\hline Taxonomic & 20 & 21 & 34 & 42 & 27 & 33 & 33 & 43 & 29 & 37 & 21 & 34 & 19 & 19 \\
\hline H' Shannon- & 1.704 & 1.936 & 1.847 & 1.732 & 1.972 & 1.885 & 2.246 & 1.930 & 1.830 & 1.930 & 0.621 & 1.804 & 1.920 & 1.342 \\
\hline J' Pielou & 0.569 & 0.636 & 0.524 & 0.463 & 0.598 & 0.539 & 0.642 & 0.513 & 0.543 & 0.535 & 0.204 & 0.511 & 0.652 & 0.456 \\
\hline
\end{tabular}

The Shannon-Wiener diversity index values presented little variation for the dry period, with only a small decrease in the $1^{\text {st }}$ down to the $2^{\text {nd }}$ order stretches and from the $5^{\text {th }}$ down to the $6^{\text {th }}$ order stretches. The rainy period also presented little variation between river stretches, except for the $3^{\text {rd }}$ (open canopy) and $5^{\text {th }}$ order stretches where an increase and a large decrease, respectively, were 
observed in the diversity values (Fig. 1). The highest values were found in the rainy period in the $3^{\text {rd }}$ order stretches (open canopy, $\mathrm{H}^{\prime}=2.246$ and closed canopy, $\mathrm{H}^{\prime}=1.972$ ), with the lowest value found in the $5^{\text {th }}$ order stretch $\left(\mathrm{H}^{\prime}=0.621\right)$ in the same period (Table 3).

The Pielou evenness values presented a pattern similar to that of the Shannon-Wiener diversity index, with higher values in the dry periods and an abrupt decrease in the $5^{\text {th }}$ order stretch (Fig. 1). The highest values were found in the $6^{\text {th }}$ order $\left(\mathrm{J}^{\prime}=0.652\right)$, and in the $3^{\text {rd }}$ order (open canopy, $\mathrm{J}^{\prime}=0.642$ ), both in the rainy period. The lowest value was found in the rainy period in the $5^{\text {th }}$ order stretch (Table 3 ).

The highest density values were found in the dry period, except for the $2^{\text {nd }}$ order stretch, where an increase was shown in the rainy period (Fig. 1). The $4^{\text {th }}\left(64,775 \mathrm{ind} / \mathrm{m}^{2}\right)$ and $5^{\text {th }}$ order $\left(90,255 \mathrm{ind} / \mathrm{m}^{2}\right)$ stretches presented highest values in the dry period (Table 3). The lowest value was found in the rainy period in the $3^{\text {rd }}$ order (closed canopy; $6,488 \mathrm{ind} / \mathrm{m}^{2}$ ).

The collectors, together with the scrapers, predominated in the benthic macroinvertebrate communities in all the river stretches, except for the $2^{\text {nd }}, 4^{\text {th }}$, and $5^{\text {th }}$ order stretches in the rainy period, where communities were predominantly filterers (Fig. 2). In these river stretches, the dominant organisms were Simuliidae (Diptera) larvae, and also filterer nymphs of Leptophlebiidae, found in lower densities (e.g., Hermanella). In the remaining stretches, the main dominant groups in both periods were the Chironomidae (Diptera) larvae and Baetidae (Ephemeroptera) nymphs (Table 3). In contrast, the shredders presented the lowest densities within the trophic groups, with abundances of lower than $15 \%$. The predators also presented low densities, except in the dry period in the first order stretch (Fig. 2) where the benthic macroinvertebrate communities were dominated by this trophic group, represented mainly by Corixidae, Veliidae, and predator larvae of Tanypodinae (Chironomidae) (Table 3). The predator density was related positively to total prey density $(\mathrm{p}<0.05 ; \mathrm{r}=0.82)$.

\section{Microbiological indicators}

The Indaiá stream presented low fecal and total coliform counts along the longitudinal gradient in both sampling periods, except for the $5^{\text {th }}$ and $6^{\text {th }}$ order stretches, which showed high counts in the rainy period (Table 4). Regarding the heterotrophic bac- terial densities, the highest values were found in the rainy period, except for the $3^{\text {rd }}$ (open canopy) and $4^{\text {th }}$ order stretches, which showed higher values in the dry period (Table 4). The assessment of yeast diversity revealed low microrganism densities along the longitudinal gradient, with eight yeast species being identified (Table 4).

\section{DISCUSSION}

In studying the structure, diversity, and functional trophic group composition of the benthic macroinvertebrate communities along the longitudinal gradient, the results indicated that, as verified in other studies, the major influences were seasonality (Oliveira et al., 1997; Bispo \& Oliveira, 1998), habitat and microhabitat diversity (Galdean et al., 2000, 2001; Callisto et al., 2001b), and the canopy cover of the riparian vegetation along the river bed (Aguiar et al., 2002).

The rainy period in the Serra do Cipó region is characterized by fierce storms that influence the whole aquatic biota, with rapid increase of water current velocity and flow, and water level rising up to 5 meters above normal values. This significant flow and water velocity increase may lead to a dislodgment of resident benthic fauna through physical disturbance of the substrate (Poff \& Ward, 1991). A decrease in taxonomic richness and total density of benthic macroinvertebrate communities was observed in the rainy period in almost all river stretches. In studying the spatial-temporal distribution of benthic macroinvertebrate communities in streams in Goiânia Ecological Park (Goiás State, Brazil), Bispo \& Oliveira (1998) found similar results: density and taxonomic richness reductions during the rainy periods.

However, the diversity and evenness indexes increased during the rainy period, except for the $1^{\text {st }}$ and $5^{\text {th }}$ order stretches. This may have happened because of food resource increase and density reductions in the benthic macroinvertebrates, which resulted in a well-structured community. The first order stretch is characterized by $100 \%$ bedrock sediment and large FPOM deposits. During the rainy period part of this material is carried off, reducing food availability and, consequently, taxonomic richness, diversity, and evenness of the benthic macroinvertebrate communities. On the other hand, in the $5^{\text {th }}$ order stretch, which presented 
lower values, reduction in the diversity and evenness values is due to the increase in densities of Simuliidae (Diptera) larvae. These organisms feed on suspended FPOM, the most abundant food resource in this river stretch during the rainy period, as pointed out by Galdean et al. (2001).
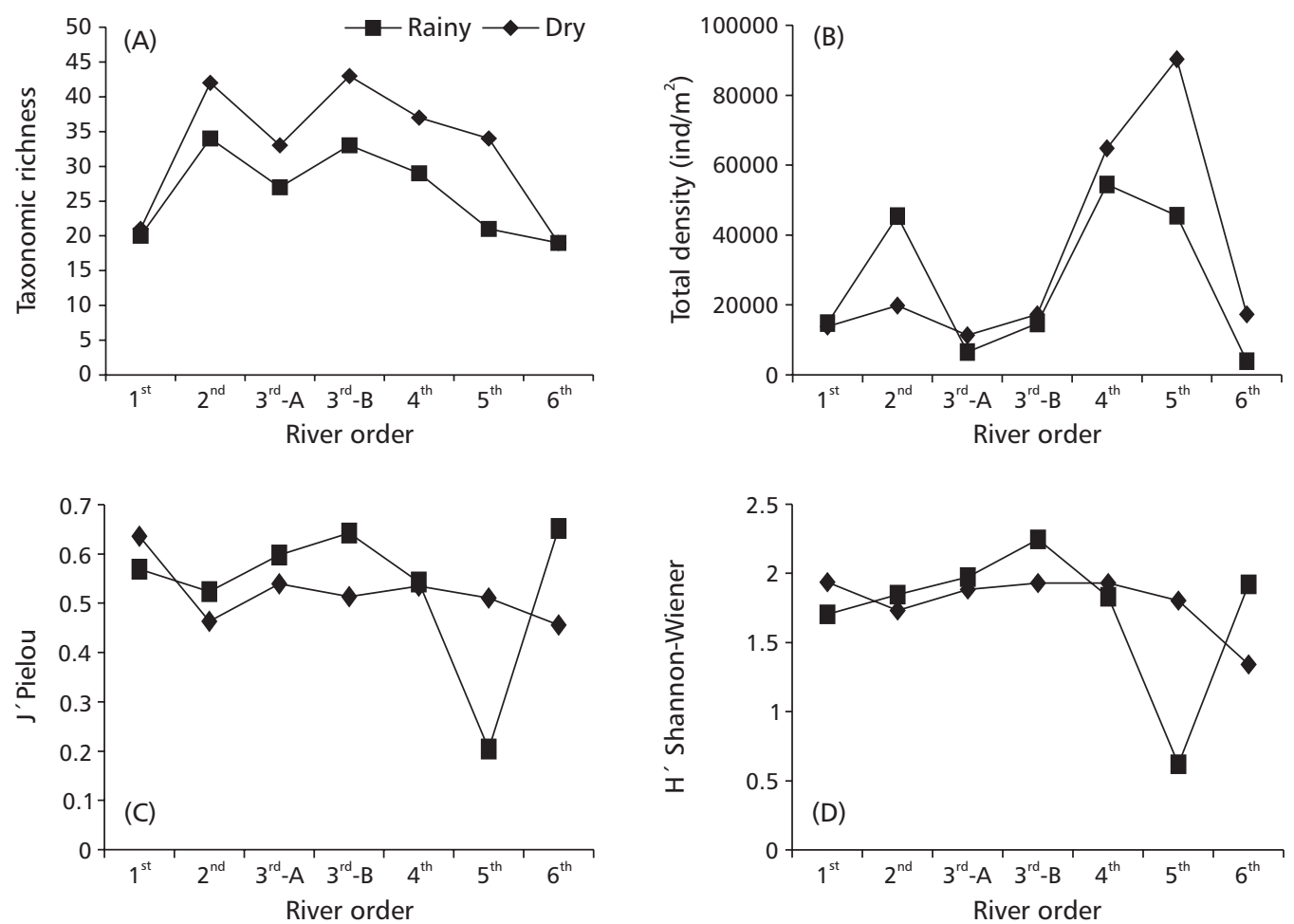

Fig. 1 - Taxonomic richness (A), total density (B), Pielou evenness index (C), and Shannon-Wiener diversity index (D) of the benthic macroinvertebrate communities during the rainy and dry periods of 2000, along the longitudinal gradient in the Serra do Cipó.
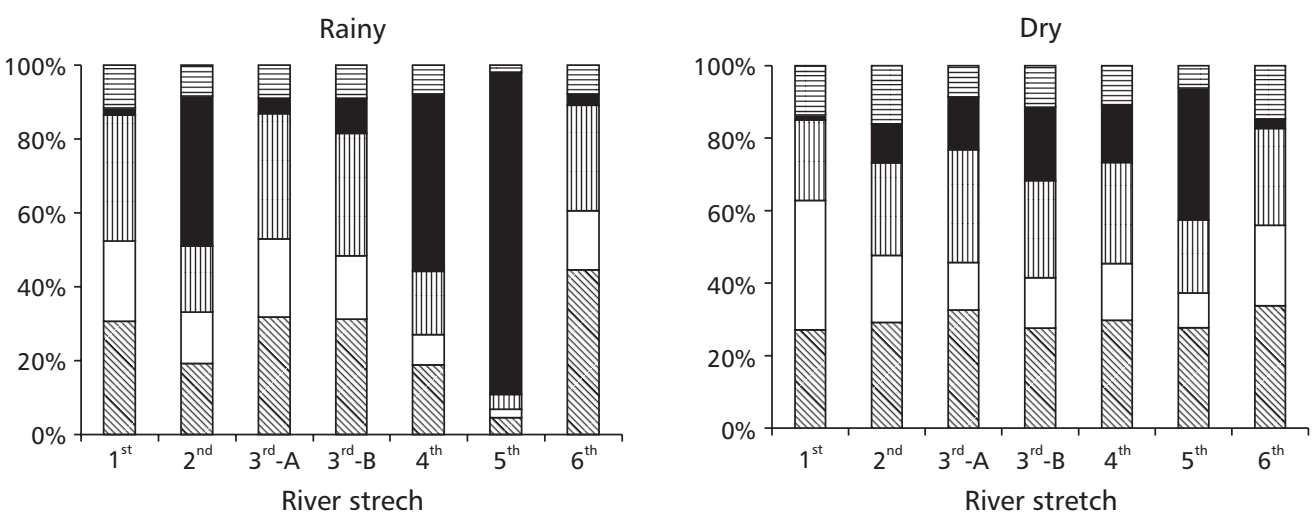

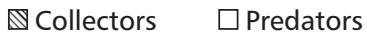

血 Scrapers

- Filterers 目Shredders

Fig. 2 - Functional trophic groups composition of the benthic macroinvertebrate communities along a longitudinal gradient in the Indaiá stream and Peixe river, in the rainy and dry periods of 2000. 
TABLE 4

Number of isolated yeast species (CFU/ml), fecal/total coliforms (MPN), and heterotrophic bacteria counts (CFU/ml) along the longitudinal gradient in the Serra do Cipó during the rainy and dry periods of 2000.

\begin{tabular}{|c|c|c|c|c|c|c|c|c|c|c|c|c|c|c|}
\hline \multirow{2}{*}{ Microorganisms } & \multicolumn{2}{|c|}{$1^{\text {st }}$ order } & \multicolumn{2}{|c|}{$2^{\text {nd }}$ order } & \multicolumn{2}{|c|}{$3^{\text {rd }}$ order $^{A}$} & \multicolumn{2}{|c|}{$3^{\text {rd }}$ order $^{B}$} & \multicolumn{2}{|c|}{$4^{\text {th }}$ order } & \multicolumn{2}{|c|}{$5^{\text {th }}$ order } & \multicolumn{2}{|c|}{$6^{\text {th }}$ order } \\
\hline & Rainy & Dry & Rainy & Dry & Rainy & Dry & Rainy & Dry & Rainy & Dry & Rainy & Dry & Rainy & Dry \\
\hline Yeasts & & & & & & & & & & & & & & \\
\hline Aurebasidium pullulans & & & & 10.3 & & & & & & & 3.3 & & 6.6 & 3.3 \\
\hline Candida sp. & & & & & & & & & & & & & 13.3 & \\
\hline C. aff sueca & & & & 3.3 & & & & & & & & & & \\
\hline C. aff utilis & & & & 3.3 & & & & & & & & & & \\
\hline Cryptococcus luteolus & & & & & & & & & & & 3.3 & & & \\
\hline Kodamaeaea ohmeri & 3.3 & & & & & & 3.3 & & 3.3 & & & & & \\
\hline Rhodotorula mucilaginosa & & 33.3 & & & & & & & & & & & & \\
\hline$R$. glutinis & & & & & & & & & & & 3.3 & & & 6.6 \\
\hline Total coliforms & 40 & 19 & 110 & 19 & 170 & 40 & 170 & 40 & 140 & 40 & 2800 & 110 & 2200 & 110 \\
\hline Fecal coliforms & $<2$ & $<2$ & 20 & $<2$ & 40 & $<2$ & 20 & $<2$ & $<2$ & 20 & 500 & 80 & 700 & 80 \\
\hline Heterotrophic bacteria & 23465.0 & 34.8 & 107664.4 & 43.3 & 82251.3 & 10231.3 & 1914.7 & 22830.5 & 56604.6 & 1354.3 & 186065.1 & 299999.8 & 155106.1 & 6299.6 \\
\hline
\end{tabular}

Collectors predominated in all river stretches and both sampling periods. But in the dry period the benthic macroinvertebrates were found to be composed mainly of collectors and scrapers while during the rainy period filterers increased considerably in numbers in all stretches, dominating the communities in the $2^{\text {nd }}, 4^{\text {th }}$, and $5^{\text {th }}$ order stretches.

Structural and compositional modifications of the benthic macroinvertebrate communities probably occur due to a change in trophic resource availability (Callisto et al., 2001b). The physical disturbance of the substrate observed in the rainy period caused the sediment to revolve, thus resuspending the FPOM deposited on the bottom. Besides that, in aquatic ecosystems pluvial increase leads to a larger input of allochthonous organic matter including both coarse and fine material (Allan, 1995). Therefore, during the rainy period an increase was found in suspended FPOM, which modified the functional trophic group composition of the benthic communities.

The shredders and predators presented low densities in both sampling periods. According to temperate region models such as the river continuum concept (Vannote et al., 1980), low-order streams located in forested areas present restricted primary production due to riparian shading. Consequently, much of the energy demand by consumers is met by allochthonous sources (Dudgeon \& Bretschko, 1995) and these stretches present high densities of organisms such as the shredders (e.g., Calamoceratidae: Trichoptera), capable of processing these coarse particulate organic matter resources.

Unlike aquatic ecosystems in temperate regions, most of the streams (specially in the upper stretches, such as $1^{\text {st }}$ and $2^{\text {nd }}$ orders) of the main watersheds in Minas Gerais State are located in mountainous areas, in which the dominant vegetation is composed by rupestrian fields. The presence of forested areas with canopies covering the river channels is determined by geomorphologic (formation and depth) and edaphic (fertility, water retention capacity) characteristics of the soil. But even in the stretches where this covering is present, an intense colonization of the sediment by mosses and algae biofilm is observed. Besides that, shredders are less abundant and present smaller biomass and secondary production if compared to temperate ecosystems (Mathuriau \& Chauvet, 2002; Ramirez \& Pringle, 1998). Regarding predator densities, it is known that these organisms are naturally less abundant in the environment, and their distribution and numbers are usually related to prey abundance (Begon et al., 1996).

\section{Water quality in the headwaters of Doce river}

The standards established by regulatory agencies that supervise recreational water use are based upon counts of the coliform group, which includes pathogenic bacteria such as Salmonella spp., present in domestic sewage. The Indaiá stream presented low densities of coliforms along the gradient, however it should be emphasized that in the $5^{\text {th }}$ and $6^{\text {th }}$ order stretches in the Peixe river a considerable increase occurred in the numbers of this microorganism. This may indicate contamination of these waters by warmblooded animal feces, an expected finding since this river is surrounded by cattle farms and small towns.

The heterotrophic bacteria counts may indicate the water-quality degradation degree, since these 
organisms are frequently associated with organic matter concentrations in water (Greenberg et al., 1998). The low heterotrophic bacteria counts along the longitudinal gradient reflect the low waterdegradation degree.

We also observed that in the $1^{\text {st }}$ to the $4^{\text {th }}$ order stretches the counts were close to the ones found in the $5^{\text {th }}$ and $6^{\text {th }}$ order stretches. This similarity is probably related to organic matter input from surrounding areas, while in the studied stretches in the Peixe river, the larger organic matter input came from non-treated effluents of farms and small towns, in addition to livestock feces.

Besides heterotrophic bacterial counts, counting and identify yeasts can indicate the eutrophication level of aquatic environments, due to the strong association of yeast with organic matter concentrations in the water (Rosa et al., 1990; Peçanha et al., 1996; Morais et al., 1996). Along the longitudinal gradient in the Indaiá stream and Peixe River low counts of these microorganisms were found, confirming the conservation level of these ecosystems.

According to a Brazilian law (number 1469, of December 29, 2000) concerning potability levels of water, all of the studied stretches in the Indaiá stream and Peixe river are inadequate for human consumption without previous treatment, due to the presence of heterotrophic bacteria. Regarding balneability, all river stretches were considered appropriate for bathing. The microbiological results of this study allow us to infer that the waters from Serra do Cipó have excellent potential for recreational use and as future sources of drinking water.

\section{Final comments}

In altitudinal lotic ecosystems the energy conversion and organic matter degradation processes are sustained mainly by algae biofilm and aquatic microbiota, are intimately related to maintenance of the physical environment, and have fundamental consequences for aquatic community structure and diversity. Studies regarding lotic ecosystem structure and functioning should include, in addition to physical characterization of the environment (canopy covering, sediment composition, forms and distribution of available organic matter, etc.) and characterization of physical (e.g., temperature, water flow) and chemical (dissolved oxygen, $\mathrm{pH}$ ) water variables, also the identification and structural characterization of the biological communities, especially the benthic macroinvertebrate and microorganism communities.
Biodiversity assessment in protected areas such as the Federal Conservation Units, which is the case of Serra do Cipó National Park, involves the study of structural characteristics and diversity of biological communities in their natural environments. The results of such studies can be used in determining restoration measures relating to these communities in anthropogenically disturbed sites. Aquatic biological communities can be preserved by creating and maintaining conservation units, implementing conservation measures outside these areas, and restorating degraded habitats (Primack \& Rodrigues, 2001).

Understanding aquatic community structural factors depends on knowing the autoecology of these organisms, as well as their use of food resources, substrate associations, and longitudinal distribution. The study of benthic macroinvertebrate community structure and composition along longitudinal gradients constitutes an important tool for aquatic ecosystem conservation in the Serra do Cipó. The importance of lateral and longitudinal dimensions of lotic ecosystems makes immediate large-scale approaches (in terms both of time and space) urgent if the elements and ecological processes supporting the biota are to be conservated and managed effectively. Besides that, use of the drainage basin as a study unit and middleand long-term monitoring program implementation are extremely necessary.

Acknowledgements - The authors are especially grateful to colleagues of the Laboratory of Benthic Ecology for field and laboratory assistance, and to L. Cota for an English revision. Logistic support provided by IBAMA, as well as financial support of CNPq (462185/00-1 and 472328/01-8), FAPEMIG (CRA - 123497), and CAPES for granting scholarships are appreciated. A. Medeiros is a Ph.D. student in the Graduation Program of Microbiology, Federal University of Minas Gerais. M. Goulart and P. Moreno are former M.Sc. students in the Graduation Program in Ecology, Conservation and Management of Wildlife, Federal University of Minas Gerais, which is maintained by the U.S. Fish and Wildlife Service. This program contributes to implementing the Convention on Nature Protection and Wildlife Preservation in the Western Hemisphere (1940) and the Ramsar Convention on Wetlands (Ramsar, Iran, 1971).

\section{REFERENCES}

AGUIAR, F. C., FERREIRA, M. T. \& PINTO, P., 2002, Relative influence of environmental variables on macroinvertebrate assemblages from an Iberian basin. Journal of North American Benthological Society, 21(1): 43-53.

ALLAN, J. D., 1995, Stream ecology: structure and function of running waters. Chapman and Hall, New York, 388p. 
BEGON, M., Harper, J. L. \& Townsend, C. R., 1996, Ecology: individuals, populations and communities. 3. ed. Blackwell Science.

BISPO, P. C. \& OLIVEIRA, L. G., 1998, Distribuição espacial de insetos aquáticos (Ephemeroptera, Plecoptera e Trichoptera) em córregos de Cerrado do Parque Ecológico de Goiânia, Estado de Goiás. Oeocologia Brasiliensis, 5: 19-33.

CAllistO, M. \& ESTEVES, F. A., 1995, Distribuição da comunidade de macroinvertebrates benthic em um ecossistema amazônico impactado por rejeito de bauxita - Lago Batata (Pará, Brasil). Oecologia Brasiliensis, 1: 335-348.

CALLISTO, M., BARBOSA, F. A. R. \& VIANNA, J. A., 1998, Qual a importância de uma coleção científica de organismos aquáticos em um projeto de biodiversidade? Anais do IV Simpósio de Ecossistemas Brasileiros. Praia, represa e mata, II(104): 432-439.

CALLISTO, M., MORETTI, M. \& GOULART, M., 2001a, Macroinvertebrates benthic com ferramenta para avaliar a saúde de riachos. Revta. Bras. Rec. Hid., 6(1): 71-82.

CALLISTO, M., MORENO, P. \& BARBOSA, F. A. R., 2001b, Habitat diversity and benthic trophic functional groups at Serra do Cipó, southeast Brazil. Braz. J. Biol., 61(2): 259-266.

CALLISTO, M., FERREIRA, W., MORENO, P., GOULART, M. \& PETRUCIO, M., 2002, Aplicação de um protocolo de avaliação rápida da diversidade de habitats em atividades de ensino e pesquisa (MG-RJ). Acta Limnol. Bras., 14: 91-98.

CARMOUZE, J. P., 1994, O metabolismo dos ecossistemas aquáticos: fundamentos teóricos, métodos de estudo e análises químicas. Ed. Edgard Blücher; FAPESP, São Paulo.

CEBALLOS, B. O. S., LIMA, E. O., KONIG, A. \& MARTINS, M. T., 1995, Spatial and temporal distribuition of fecal coliforms, coliphages, moulds and yeasts in freshwater at semi-arid tropic northeast region in Brazil (Paraíba State). Rev. Microbiol., 26: 177-181.

CRANSTON, P. S., 1996, Identification Guide to the Chironomidae of New South Wales. CSIRO Division of Entomology. AWT - Australian Water Technologies Insight.

DOMÍNGUEZ, E., HUBBARD, M. D., PESCADOR, M. L. \& MOLINERI, C., 2001, Epheroptera. In: H. R. Fernández \& E. Domínguez (eds.), Guía para la determinación de los artrópodos bentónicos sudamericanos. Ed. Universidad Nacional de Tucumán, pp. 17-54.

DUDGEON, D., 1994, Research strategies for the conservation and management of tropical Asian streams and rivers. International Journal of Ecology \& Environmental Scinces, 20: 255-285.

DUDGEON, D. \& BRETSCHKO, G., 1995, Allochthonous inputs and land-water interactions in seasonal streams: tropical Asia and temperate Europe. In: F. Schiemer \& K. T. Boland (eds.), Perspectives in Tropical Limnology. SPB Academic Publishing, The Hague, pp. 161-179.

EPLER, J. H., 2001, Identification manual for the larval chironomidae (diptera) of north and south Carolina. North Carolina Department of Environmental and Natural Resources - Division of Water Quality. North Carolina.
GALDEAN, N., CALLISTO, M. \& BARBOSA, F. A. R., 2000 Lotic ecosystems of Serra do Cipó, southeast Brazil: water quality and a tentative classification based on the benthic macroinvertebrate community. Aquatic Ecosystem Health and Management, 3: 545-552.

GALDEAN, N., CALLISTO, M. \& BARBOSA, F. A. R., 2001, Biodiversity assessment of benthic macroinvertebrates in altitudinal lotic ecosystems of Serra do Cipó (MG, Brazil). Braz. J. Biol., 61(2): 239-248.

GALVÃO, M. V. \& NIMER, E., 1965, Clima, pp. 91-139. In: Geografia do Brasil-Grande Região Leste, v. 5. IBGE, Rio de Janeiro.

GOLTERMAN, H. L., CLYMO, R. S. \& OHNSTAD, M. A. M., 1978, Methods for chemical analysis of freshwaters. Blackwell Scientific Publications, 210p.

GREENBERG, A. E., CLESCERI, L. S. E. \& EATON, A. D., 1998, Standard methods for the examination of water and waste water. 18. ed. A.P.H.A., Washington.

HAGLER, A. N., MENDONÇA-HAGLER, L. C., SANTOS, E. A., FARAGE, S., SILVA FILHO, J. B. \& SCHRANK, A., 1986, Microbiol pollution indicators in Brazilian tropical and subtropical; marine surface water. Sci. Total. Environ., 58: 151-160.

KURTZMAN, C. P. \& FELL, J. W., 1998, The yeast: a taxonomic study. 4. ed. Elsevier, Amsterdam.

LUTTERBACK, M. T. S., VAZQUEZ, J. C., PINET, J. A., ANDREATA, J. V. \& DA-SILVA, A. C., 2001, Monitoring and spatial distribution of heterotrophic bacteria and fecal coliforms in the Rodrigo de Freitas lagoon, Rio de Janeiro, Brazil. Braz. Arch. Biol. Technol., 44(1).

MACKERETH, F. J. H., HERON, J. \& TALLING, J. F., 1978, Water Analysis: some revised methods for limnologists. Freshwater Biological Association, Windermere, 120p.

MAGURRAN, A. E., 1991, Ecological diversity and its measurement. Chapman and Hall, London, 178p.

MATHURIAU, C. \& CHAUVET, E., 2002, Breakdown of leaf litter in a neotropical stream. J. N. Am. Benthol. Soc., 21(3): 384-396.

MORAIS, P. B., RESENDE, M. A., ROSA, C. A. \& BARBOSA, F. A. R., 1996, Occurrence and dial distribution of yeast in a Paleo-karstic lake of Sotheastern Brazil. Rev. Microbiol., 27: $182-188$.

OLIVEIRA, L. G., BISPO, P. C. \& SÁ, N. C., 1997, Ecologia de assembléias de insetos bentônicos (Ephemeroptera, Plecoptera e Trichoptera), em córregos do parque ecológico de Goiânia, Goiás, Brasil. Revta. Bras. Zool., 14(4): 867-876.

PEÇANHA, M. P., PAGNICCA, F. C., RUGANI, C. A. \& NEVES, F. A., 1996, Yeast and other parameters of pollution of Ribeirão Claro stream in Rio Claro, São Paulo. Rev. Microbiol., 27: 177-181.

PÉREZ, G. P., 1988, Guía para el studio de los macroinvertebrados acuáticos del departamento de Antioquia. Editorial Presencia Ltda., Bogotá, 217p. 
PESCADOR, M. L., 1997, General Ecology of Mayflies: adaptations, reproductive strategies and trophic categories. In: Taller internacional sobre sistemática y bioecología de Ephemeroptera como bioindicador de la calidad del agua. Santiago de Cali, Colombia, 10p.

POFF, N. L. \& WARD, J. V., 1991, Drift responses of benthic invertebrates to experimental stream flow variation in a hydrologically stable stream. Canadian. Journal of Fisheries and Aquatic. Sciences, 48: 1926-1936.

PRIMACK, R. B. \& RODRIGUES, E., 2001, Biologia da conservação. Gráfica e editora Midiograf, Londrina, 327p.

RAMIREZ, A. \& PRINGLE, C. M., 1998, Structure and production of a benthic insect assemblage in a neotropical stream. J. N. Am. Benthol. Soc., 17(4): 443-463.

ROSA, C. A., REZENDE, M. A., BARBOSA, F. A. R., MORAIS, P. B. \& FRANZOT, S. P., 1995, Yeast diversity in a mesotrophic lake on the karstic plateau of Lagoa Santa, MGBrazil. Hidrobiologia, 308: 103-108.

ROSA, C. A., REZENDE, M. A., FRANZOT, S. P., MORAIS, P. B. \& BARBOSA, F. A. R., 1990, Distribuição de leveduras e coliformes em um lago do Karst do planalto de Lagoa Santa, MG, Brasil. Rev. Microbiol., 21: 19-24.
ROSENBERG, D. M. \& RESH, V. H., 1993, Introduction to freshwater biomonitoring and benthic macroinvertebrates. In: D. M. Rosenberg \& V. H. Resh. Freshwater biomonitoring and benthic macroinvertebrates. Chapman and Hall, New York, pp.1-9.

TUNDISI, J. G. \& BARBOSA, F. A. R., 1995, Conservation of aquatic ecosystems: present status and perspectives. In: J. G. Tundisi, C. F. M. Bicudo \& T. Matsumura-Tundisi (eds.), Limnology in Brazil. BAS, BLS, São Paulo.

VANNOTE, L. R., MINSHALL, G. W., CUMMINS, K. W., SEDELL, J. R. \& CUSHING, C. E., 1980, The river continuum concept. Can. J, Fish. Aquat. Sci., 37: 130-137.

WARD, J. V., 1992, Aquatic insect ecology: 1. Biology and habitat. John Wiley \& Sons, New York, 438p.

WIGGINS, G. B., 1977, Larvae of the North American caddisfly genera (Trichoptera). University of Toronto Press, Toronto, $401 \mathrm{p}$.

YARROW, D., 1998, Methods for the isolation, maintenance, classification and identification of yeasts. In: C. P. Kurtzman $\&$ J. W. Fell (eds.), The yeasts: a taxonomic study, $4^{\text {th }}$ ed. Elsevier Science B. V., Amsterdam, pp. 77-100. 